\title{
Generation and Characterization of Isolates of Peronophythora litchii Resistant to Carboxylic Acid Amide Fungicides
}

\author{
Hancheng Wang, Haiyan Sun, Gerd Stammler, Jianxia Ma, and Mingguo Zhou
}

First, second, and fifth authors: College of Plant Protection, Nanjing Agricultural University, Key Laboratory of Monitoring and Management of Crop Diseases and Pest Insects, Ministry of Agriculture, Nanjing 210095, P.R. China; third author: BASF SE, 67117 Limburgerhof, Germany; and fourth author: BASF (China) Co. Ltd., Shanghai, 200001, P.R. China.

Accepted for publication 25 January 2010.

\begin{abstract}
Wang, H. C., Sun, H. Y., Stammler, G., Ma, J. X., and Zhou, M. G. 2010. Generation and characterization of isolates of Peronophythora litchii resistant to carboxylic acid amide fungicides. Phytopathology 100:522527.

Four isolates of Peronophythora litchii with resistance to carboxylic acid amide (CAA) fungicides were selected on fungicide-amended agar. These isolates had various levels of resistance, as evidenced by their resistance factor (RF), which is the $50 \%$ effective concentration $\left(\mathrm{EC}_{50}\right)$ value of a particular isolate divided by that of the wild-type parent. RF values to dimethomorph for the four isolates were $15,24,141$, and $>1,500$. Resistance was stable for two isolates, while the $\mathrm{EC}_{50}$ values

medium. Cross-resistance occurred with all CAAs tested here (dimethomorph, mandipropamid, flumorph, and pyrimorph), but not with strobilurins (azoxystrobin and famoxadone) or other fungicides (metalaxyl, cymoxanil, and mancozeb). Studies on fitness parameters (mycelial growth, sporulation, spore germination, zoospore formation, aggressiveness, and temperature tolerance) in the parent wild-type and resistant isolates demonstrated that penalties in different parameters may be associated with CAA resistance, depending on the isolate. These studies show that Peronophythora litchii is able to express CAA resistance under laboratory conditions but it is not known if resistant strains could become established in the field and sensitivity monitoring studies are recommended.
\end{abstract} decreased for the other two after repeated subculturing on fungicide-free
Peronophythora litchii Chen ex Ko et al. causes downy blight on lychee (litchi) (Litchi chinensis Sonn.), which frequently occurs in the subtropical parts of southern China, India, Thailand, and Vietnam. The pathogen, which is a transitional species between Phytophthora and Peronospora $(6,23,27)$, attacks fruit, panicles, and new shoots, causing panicle rot and withering and watery brown spots on fruits. The pathogen also causes significant post-harvest losses. Control of the disease is based on an integration of several cultural methods with the use of fungicides. In the 1990s, multisite inhibitors, such as mancozeb and chlorothalonil, and the target site-specific phenylamide metalaxyl were used to control this disease (24). A novel fungicide group, the carboxyl acid amide (CAA) fungicides, was first registered in 1996 in China for the control of litchi downy blight (25). The mode of action of CAAs has not yet been fully elucidated, but hypotheses include inhibition of phospholipid biosynthesis (12) and interference with cell wall deposition $(2,7,9,15-17)$.

The CAA fungicide dimethomorph is used extensively in the world against different plant-pathogenic oomycetes, such as Pseudoperonospora cubensis, Plasmopara viticola, and Phytophthora infestans. After intensive use of CAA fungicides for more than 15 years against oomycete pathogens, no resistant field isolates have been detected in Phytophthora infestans populations (FRAC CAA Working Group, www.frac.info) (8), but CAAresistant isolates have been detected in populations of Plasmopara viticola in some European regions and there are also reports of CAA resistance in Pseudoperonospora cubensis from a few trial site locations in South Korea, Israel, the United States (FRAC CAA Working Group), and China (28). In recent moni-

Corresponding author: M. G. Zhou; E-mail address: mgzhou@njau.edu.cn

doi:10.1094/PHYTO-100-5-0522

(c) 2010 The American Phytopathological Society toring studies no resistant isolates have been reported in Peronophythora litchii populations (FRAC CAA Working Group2008 Minutes).

Artificial mutants resistant to CAAs have been generated in Phytophthora infestans, Phytophthora capsici, and Phytophthora parasitica by exposure of mycelia $(5,21,26)$, sporangia $(18,19)$, or cystospores (2) to UV light or NMU ( $N$-nitrosomethylurea). However, CAA-resistant mutants of Phytophthora spp. have never been identified in the field.

The objectives of the present study were to (i) generate isolates of Peronophythora litchii resistant to CAA fungicides, (ii) characterize these isolates for their resistance factor (RF), stability of resistance and cross-resistance within the CAA fungicides and other fungicides with oomycete activity, and (iii) determine if the sensitivity changes are associated with fitness penalties in mycelial growth, spore production, aggressiveness, and temperature tolerance.

\section{MATERIALS AND METHODS}

Fungal strains and culture conditions. One isolate of Peronophythora litchii, with wild-type sensitivity to the CAA fungicide dimethomorph, obtained from the Department of Plant Pathology of South China Agricultural University, was used as a parent strain to generate CAA-resistant isolates. Seven other dimethomorph-sensitive single-sporangium isolates of Peronophythora litchii were collected from diseased fruits from Guangxi Province of China in 2007, which served as sensitive reference isolates in cross-resistance tests. All isolates were grown and maintained on lima bean agar medium (LBA, $60.0 \mathrm{~g} \mathrm{liter}^{-1}$ of lima beans boiled for $1 \mathrm{~h}, 16.0 \mathrm{~g} \mathrm{liter}^{-1}$ of agar) in a controlled climate cabinet at $25^{\circ} \mathrm{C}$ in darkness. For long-term storage, the isolates were maintained in glass tubes on LBA medium at $25^{\circ} \mathrm{C}$ in the dark and single tip transfers were made every 3 months. 
Fungicides. Stock fungicide preparations were obtained by dissolving technical-grade dimethomorph (a.i. 98.3\%, BASF China Co., Ltd., Shanghai, China), mandipropamid (a.i. 96.1\%, Syngenta China Co., Ltd., Shanghai, China), flumorph (a.i. 92.5\%, Shenyang Research Institute of Chemical Industry, Shenyang, China), pyrimorph (a.i. 98\%, Gengyun Chemicals Jiangsu, Zhenjiang, China), azoxystrobin (a.i. 93\%, Syngenta China Co.), famoxadone (a.i. 98\%, DuPont China Co., Ltd., Shanghai, China), metalaxyl (a.i. 98\%, Baolin Chemicals Jiangsu, Nantong, China), and cymoxanil (a.i. 98\%, Liming Chemicals Jiangsu, Nanjing, China) in methanol. Mancozeb (a.i. 80\%, DuPont China Co.) was suspended in distilled water. Stock preparations were diluted in water as required and stored at $4{ }^{\circ} \mathrm{C}$ in darkness. The methanol concentration never exceeded $0.5 \%$ of the test solution. This concentration did not affect mycelial growth, germination of cystospores or sporangia of Peronophythora litchii (data not shown). Controls always contained the same methanol concentration as test samples in the experiments.

Generation of CAA-resistant isolates. Selection for resistance involved two steps. First, 216 mycelial plugs ( $5 \mathrm{~mm}$ in diameter) were excised randomly from 4-day-old whole colonies of Peronophythora litchii growing on LBA and placed upside down on fresh LBA plates containing $0.25 \mathrm{mg} \mathrm{liter}^{-1}$ of dimethomorph. After incubation at $25^{\circ} \mathrm{C}$ for more than 30 days in darkness, the survivors were transferred to LBA plates containing $0.3 \mathrm{mg} \mathrm{liter}^{-1}$ of dimethomorph for identification. The second step of selection was carried out with low-resistant survivors obtained in the first cycle, which were subsequently subjected to screening as described above (200 mycelial plugs for 30 days), except that in this case LBA plates contained $20 \mathrm{mg} \mathrm{liter}^{-1}$ of dimethomorph.

Sensitivity tests. For both sensitive and resistant isolates of Peronophythora litchii, individual agar disks $(5 \mathrm{~mm})$ were removed from the edge of actively growing cultures and placed face up on the center of a petri dish containing LBA amended with different concentrations of fungicides. Final concentrations were $0,0.0125,0.025,0.05,0.1,1,10$, and $100 \mathrm{mg} \mathrm{liter}^{-1}$ for azoxystrobin and famoxadone; $0,0.0625,0.125,0.25,0.5,1$, and $2 \mathrm{mg}$ liter $^{-1}$ for metalaxyl; $0,1.25,2.5,5,10$, and $20 \mathrm{mg} \mathrm{liter}{ }^{-1}$ for mancozeb; and $0,2.5,5,10,20,40$, and $80 \mathrm{mg}$ liter $^{-1}$ for cymoxanil. For sensitive reference isolates, final concentrations were $0,0.05,0.08,0.11,0.14,0.17$, and $0.2 \mathrm{mg} \mathrm{liter}^{-1}$ for dimethomorph; $0,0.0013,0.0025,0.005,0.008,0.01$, and $0.02 \mathrm{mg} \mathrm{liter}^{-1}$ for mandipropamid; $0,0.0625,0.125,0.25,0.5$, and $1.0 \mathrm{mg} \mathrm{liter}^{-1}$ for flumorph; and $0,0.05,0.10,0.20,0.40$, and $0.80 \mathrm{mg} \mathrm{liter}^{-1}$ for pyrimorph. For sensitivity tests of the resistant isolates, final concentrations of all four CAA fungicides were 0 , $0.78,1.56,3.13,6.25,12.5,25,50$, and $100 \mathrm{mg} \mathrm{liter}^{-1}$. For each fungicide at each concentration, three replicates were used. The experiments were replicated twice for each isolate. After incubation at $25^{\circ} \mathrm{C}$ in the dark for 6 days, mycelial growth of each plate was assessed as the mean of two colony diameters taken perpendicularly to each other; the diameter of the mycelial plug (5 mm) was subtracted.

Stability of resistance. Stability of resistance in vitro was analyzed by culturing resistant isolates on LBA plates without fungicides. The cultures were transferred weekly onto LBA plates and incubated at $25^{\circ} \mathrm{C}$ in the dark. For each isolate, three replicate plates were used and in total the isolates were recultured for 11 generations. Afterwards, $50 \%$ effective concentration $\left(\mathrm{EC}_{50}\right)$ values were determined with the method described below.

Fitness studies. Resistant isolates of Peronophythora litchii were compared with the wild-type parent strain for mycelial growth, sporulation (in vitro and in vivo), sporangial differentiation into zoospores, germination of cystospores, direct germination of sporangia, aggressiveness, and temperature tolerance. All experiments were repeated. For mycelial growth, four 5-mm mycelial agar plugs from each isolate were transferred to the center of LBA plates for radial growth measurements. After
6 days of incubation at $25^{\circ} \mathrm{C}$ in darkness, the colony diameter of each of the four replicate plugs of each isolate was measured as the mean of two colony diameters taken perpendicularly.

For evaluation of sporangia, zoospores, and cystospores, sporangia were harvested in $1 \mathrm{ml}$ of distilled water in a $2-\mathrm{ml}$ reaction tube from agar plugs cut approximately $5 \mathrm{~mm}$ from the colony margin with a cork borer from 5-day-old cultures growing on LBA. Differentiation of sporangial cytoplasm into zoospores was assessed by incubation of the sporangial suspension $\left(10^{4}\right.$ sporangia liter $\left.{ }^{-1}\right)$ in $1 \mathrm{ml}$ of distilled water in a 2-ml reaction tube for $2 \mathrm{~h}$ at $16^{\circ} \mathrm{C}$ in the dark. Zoospore release started after 30 min incubation and maximum zoospore numbers occurred $2 \mathrm{~h}$ later. A sporangium was scored as released if the sporangium was empty, which was easy to determine microscopically. Empty sporangia were quantified on microscope slides at three sites by counting 100 sporangia per site and the percentages of released sporangia per site were calculated.

Cystospore germination was analyzed by incubation of cystospore suspensions at $16^{\circ} \mathrm{C}$ in 2-ml reaction tubes for $4 \mathrm{~h}$, and sporangia germination by incubation of a sporangia suspension on LBA plates at $30^{\circ} \mathrm{C}$ in darkness for $8 \mathrm{~h}$. Cystospores were collected with a micropipette from a 4-h-old zoospore suspension that had been produced as described above. Sporangia were collected from agar plugs as described above. Germ tube emergence of cystospore and sporangia was quantified on microscope slides and on LBA plates at three sites, respectively, by counting 100 spores per site with a microscope. A spore was scored as germinated if the germ tube had reached at least full length of the spore.

Sporulation in vitro. For quantification of the sporangial production a previously described method (4) was used with modifications. Six colonized agar plugs $(5 \mathrm{~mm})$ were excised from each of three replicate plates. Three plugs were harvested at approximately $2 \mathrm{~mm}$ distance from the colony margin and another three from approximately $2 \mathrm{~mm}$ distance from the edge of the initial plug. The six plugs were put into a single 2-ml reaction tube with $1.0 \mathrm{ml}$ of sterile distilled $\mathrm{H}_{2} \mathrm{O}$ and agitated for $20 \mathrm{~s}$ with a laboratory vortex to dislodge the sporangia. Sporangia were quantified with a hemacytometer and the number per square centimeter of colony area was calculated.

Aggressiveness. Aggressiveness of each isolate was examined on litchi fruits. Twelve fresh ripe fruits (cv. Feizixiao) were tested per isolate. Fruits were washed three times with sterile $\mathrm{H}_{2} \mathrm{O}$ and dried with filter paper. A superficial wound was made at the middle of each fruit. Then, a mycelial plug $(5 \mathrm{~mm})$ was taken with a cork borer from the margin of a 4-day-old colony on LBA medium and placed inverted on the wound. After inoculation, fruits were incubated in a climate chamber $\left(30^{\circ} \mathrm{C}\right.$, relative humidity $>80 \%$, darkness) for $72 \mathrm{~h}$. Lesion sizes of infection were measured as the mean of two lesion diameters taken perpendicularly, and the areas of diseased lesions were calculated. For each isolate, all areas were combined and the average value was presented.

Sporulation in vivo. For sporangial production in vivo, the mass of sporangia produced on litchi fruits was determined. Fruits were inoculated as described above and, after $72 \mathrm{~h}$ incubation, lesion sizes were measured as the mean of two lesion diameters taken perpendicularly, and the areas of diseased lesions were calculated. Sporangia were collected by washing lesions with 3 $\mathrm{ml}$ of distilled water; sporangia were dislodged with the help of a sterile brush and the number of sporangia was counted in a hemacytometer. Four replicate droplets were counted and the number of sporangia per square centimeter of diseased fruit surface was calculated.

Temperature tolerance. The parent culture and resistant isolates were incubated on LBA plates at $18,20,25,30$, and $37^{\circ} \mathrm{C}$ for 7 days, and at $10^{\circ} \mathrm{C}$ for 37 days, respectively, and mycelial growth of each isolate was determined. For each isolate, three LBA plates were used at each temperature. 
Data analysis. Data from repeated experiments were combined for analysis because variances between experiments were homogeneous. All data were processed with the SIGMASTAT Statistical Software Package (SPSS Science, version 11). EC 50 values were calculated from the sensitivity tests described above from the fitted regression line of the log-transformed percent inhibition plotted against the log-transformed fungicide concentration (3). Resistance factors (RF) were calculated for all resistant isolates using the following formula: $\mathrm{RF}=\mathrm{EC}_{50} \mathrm{X} / \mathrm{EC}_{50} \mathrm{WT}$, where $\mathrm{EC}_{50} \mathrm{X}$ is the $\mathrm{EC}_{50}$ value of the strain being examined, and $\mathrm{EC}_{50} \mathrm{WT}$ is the $\mathrm{EC}_{50}$ value of the wild-type strain of that isolate. Cross-resistance was analyzed using regression analysis (22).

\section{RESULTS}

Selection of isolates of Peronophythora litchii resistant to dimethomorph. Two resistant isolates (Yg6 and $\mathrm{Yg} 9$ ) were obtained from the first selection step and another two resistant isolates (Yg6-1 and Yg6-3) by subsequent culturing of the isolate Yg6 on LBA plates amended with $20 \mathrm{mg} \mathrm{liter}^{-1}$ of dimethomorph (Table 1).

Sensitivity tests. Four resistant isolates (Yg6, Yg9, Yg6-1, and Yg6-3) and seven wild-type isolates were tested for their sensitivity to CAAs and fungicides with different modes of action. All resistant isolates showed reduced sensitivities to the four CAA fungicides compared with the parent wild-type isolate of Peronophythora litchii (Fig. 1). Cross-resistance between dimethomorph and three other CAAs (mandipropamid, flumorph and pyrimorph) was found (regression $R^{2}$ coefficients were 0.9143 , 0.9746 and 0.8623 , respectively) (Fig. 1), but not with azoxystrobin, famoxadone, metalaxyl, and mancozeb (regression $R^{2}$ coefficients were $0.0376,0.0006,0.0061$, and 0.0013 , respectively) (Fig. 2). Cymoxanil did not inhibit any isolate including the sensitive ones in this assay $\left(\mathrm{EC}_{50}\right.$ values $\left.>200 \mathrm{mg} \mathrm{liter}^{-1}\right)$.

Stability and level of resistance. Resistance of the isolates Yg6 and Yg9 was stable even after 11 successive subcultures on LBA media, while for Yg6-1 and Yg6-3, the EC $_{50}$ values decreased after culture without selection pressure (Table 1). After 11 subcultures, the $\mathrm{Yg}$ 9 had the highest resistance to CAA fungicides, with RF >1,500 for dimethomorph followed by Yg6-1, $(\mathrm{RF}=141), \mathrm{Yg} 6-3$, and Yg6 (with RF values of 24 and 15, respectively).

Fitness studies. Mycelial growth of the resistant isolates Yg6 and, moreover, $\mathrm{Yg} 9$ was significantly reduced compared with the parent isolate or the resistant isolates Yg6-1 and Yg6-3 (Table 2). In vitro and in vivo sporulation was reduced for all resistant isolates and the reduction was statistically significant for Yg6, Yg6-1, and Yg6-3 (Table 2). A decreased rate of germinated sporangia and cystospores was also associated with resistance and in most cases was statistically significant (Table 2). Zoospore formation was also slightly reduced for all resistant isolates compared with their parent isolate (Table 2).

The in vivo experiment showed that all resistant isolates were able to infect the fruit tissue (Fig. 3), but Yg6, Yg6-1, and Yg6-3 were less aggressive than the parent isolate (Table 2). In tem- perature tolerance tests the isolates Yg6, Yg6-1, and Yg6-3 showed no reduced growth at all tested temperatures, but the isolate with the highest RF value, Yg9, grew significantly slower than all others, including the parent isolate, at all temperatures tested (Table 3 ). At $37^{\circ} \mathrm{C}$, no growth of any isolate was observed.
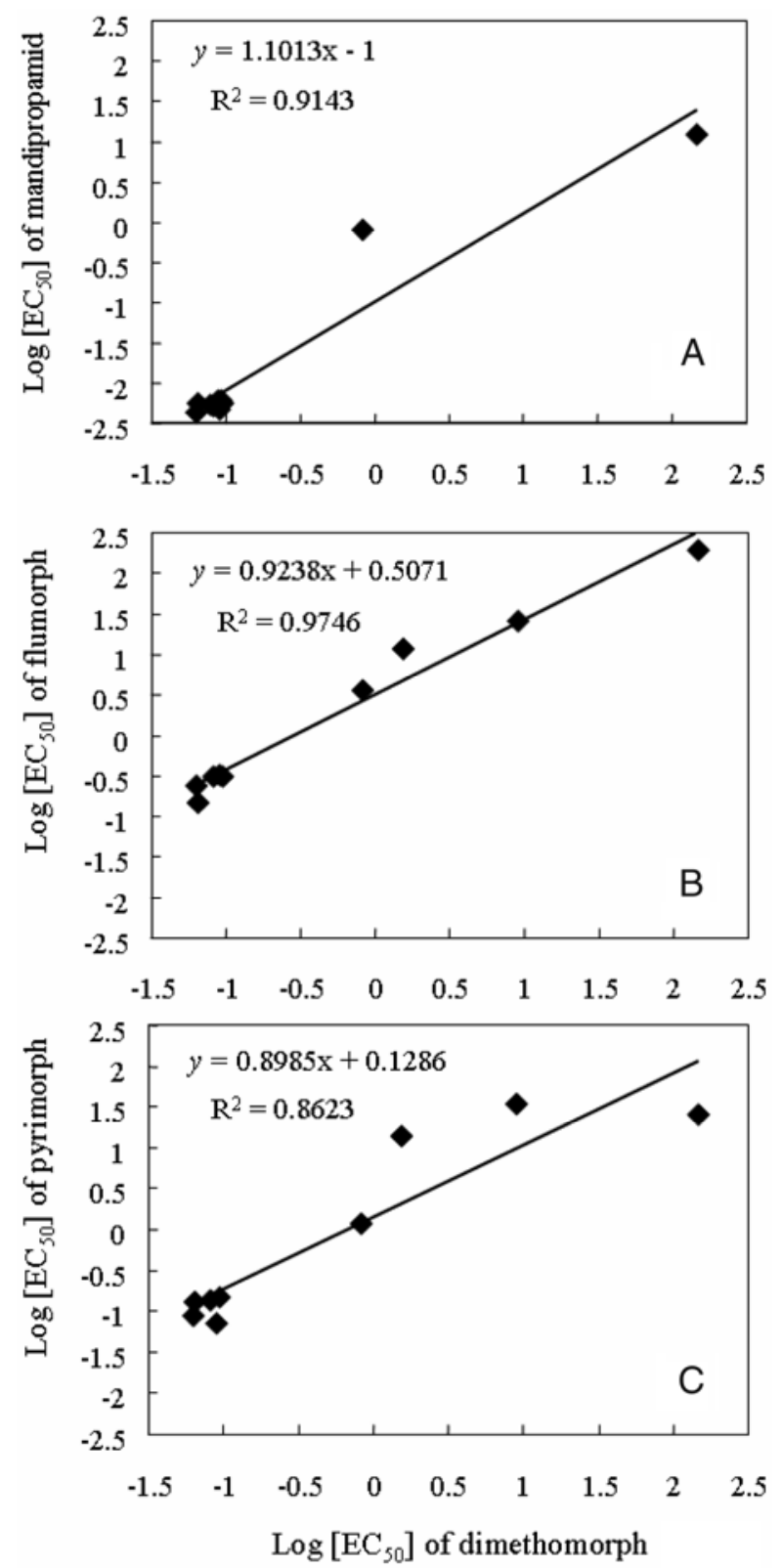

Fig. 1. Cross-resistance relationships between the carboxylic acid amide fungicides $\mathbf{A}$, dimethomorph and mandipropamid, $\mathbf{B}$, flumorph, and $\mathbf{C}$, pyrimorph in Peronophythora litchii isolates determined in mycelial growth tests on fungicide-amended agar.

TABLE 1. Sensitivity to dimethomorph of fungicide-resistant isolates of Peronophythora litchii and the parent isolate before and after 11 consecutive subculture cycles on fungicide-free medium

\begin{tabular}{lccccc}
\hline Isolate & ${\text { Initial } \mathrm{EC}_{50} \mathrm{x}}^{\mathrm{x}}$ & Initial EC $_{90} \mathrm{y}$ & Final EC $_{50} \mathrm{x}^{\mathrm{x}}$ & Final EC $_{90}{ }^{\mathrm{y}}$ & Resistance factor $^{\mathrm{z}}$ \\
\hline Parent & $0.065 \pm 0.001$ & $0.13 \pm 0.005$ & $0.064 \pm 0.001$ & $0.13 \pm 0.007$ & - \\
Yg6 & $0.86 \pm 0.02$ & $15.05 \pm 0.4$ & $0.93 \pm 0.03$ & $15.43 \pm 0.3$ & 15 \\
Yg9 & $>100$ & $98 \pm 0.6$ & $>100$ & $>100$ & $65 \pm 0.7$ \\
Yg6-1 & $18 \pm 0.3$ & $>100$ & $1.55 \pm 0.1$ & $5.82 \pm 0.3$ & $>1,500$ \\
Yg6-3 & $90 \pm 0.4$ & & 9.4 & 24 \\
\hline
\end{tabular}

${ }^{x}$ Effective concentration at $50 \%\left(\mathrm{EC}_{50}\right)$ values in $\mathrm{mg}$ liter $^{-1}$.

${ }^{y}$ Effective concentration at $90 \%\left(\mathrm{EC}_{90}\right)$ values in $\mathrm{mg} \mathrm{liter}^{-1}$.

${ }^{\mathrm{z}}$ The final $\mathrm{EC}_{50}$ value of resistant isolates divided by the final $\mathrm{EC}_{50}$ value of the parental isolate. 


\section{DISCUSSION}

During the last decade, chemical control of litchi downy blight in Asia-Pacific has been based mainly on the use of dimethomorph (13). However, no CAA-resistant isolates have been reported so far. This study was aimed at investigating the potential for Peronophythora litchii to develop resistance to CAA fungicides.

Cross-resistance experiments confirmed cross-resistance within the CAA fungicide group, but no cross-resistance with other oomycete fungicides was observed. This indicates that the resistance mechanisms expressed in these isolates are specific to CAA fungicides. Different levels of resistance may indicate different mechanisms or may suggest that a particular mechanism is more active in the more resistant $\mathrm{Yg}$ 9 than in the other three resistant isolates. Since resistance was specific for CAA fungicides, it is more probable that the mechanisms are related to the target protein of CAAs, rather than to other less specific mechanisms such as detoxification by metabolization or reduced intracellular accumulation by overexpression of efflux transporter molecules. Different target site mutations may cause different levels of sensitivity, as has been reported for strobilurins (10), triazoles (20), or benzimidazoles (14). But also different resistance mechanisms at the target, such as target site mutation and target site overexpression could result in different resistance levels.

The CAA-resistant isolates occurred frequently in our assay, as they were found in a relatively small sample size. No mutagenic agents were used in order to avoid mutations in other genes which could cause fitness penalties and erroneously lead to the conclusion that resistance is connected with fitness penalties. However, the frequency of resistant isolates might be drastically increased under fungicide stress conditions (28) and therefore this may represent an explanation for the relatively frequent occurrence of resistant strains in our study. Another explanation for the fact that resistant isolates were generated in a small sample might be a transient adaptation (e.g., by upregulation of detoxification mechanisms), which is not genetically manifest. Transient adaptation mechanisms are most likely energy-dependent and should have been down-regulated after a certain period of cultivation without exposure to CAAs. In our experiments resistance was stable for the isolate with the highest RF-value ( $\mathrm{Yg} 9)$ even after 11 transfers (and meanwhile after many more cycles of cultivation on nonfungicide-amended agar medium for more than one additional year), which suggests that the resistance mechanism of $\mathrm{Yg} 9$ is caused rather by a genetic mutation than by a transient adaptation. Whether the resistance mechanisms of the isolates concerned are based on adaptation or genetic mutations could be elucidated by inheritance studies with resistant and sensitive isolates and analysis of the $F_{1}$ and $F_{2}$ generation.

Although the genetic basis of resistance to CAAs in Peronophythora litchii is not known, resistance to CAAs in Plasmopara viticola was found to be caused by one or two recessive nuclear genes (11). With sexual reproduction, generally only part of the progeny will carry the trait of resistance (depending on dominance and zygosity); while with asexual reproduction, all progeny have the trait. Peronophythora litchii is homothallic (self-fertile), and undergoes many short disease cycles per season with a high dispersal of spores over time and space and a random sexual recombination in the disease cycle (1). It could be classified as a medium-high risk pathogen (24).

Survival of resistant isolates in the field is a result of the selection pressure of the fungicide on the one hand and fitness penalties which might be correlated with the expression of the resistance mechanism on the other. Our studies showed that the isolates with reduced sensitivity were also less fit for one or more of the parameters we measured. However, the experiments were carried out under more or less optimal infection and growth conditions without direct intraspecies competition between sensitive and mutated strains or interspecies competition with bacteria, fungi or other organisms. Survival in the field is influenced by numerous factors and it is questionable if they could all be simulated in the laboratory or in greenhouse studies. Several reports on CAA resistance in laboratory-generated mutants of Phytophthora infestans are available $(2,19,21,29)$, but fitness studies on these mutants did not support a hypothesis of development of resistance in the field. CAA-resistant mutants of Phytophthora infestans were obtained by UV irradiation at a frequency of an approximately $1 \times 10^{-7}$ and with high RF values (400 to 1,333 for iprovalicarb and benthiavalicarb, respectively) (29). Mutants with $\mathrm{RF}$ values $>20$ to dimethomorph were generated after ethidium bromide/UV light mutagenesis $(19,21)$ and mutants with $\mathrm{RF}$
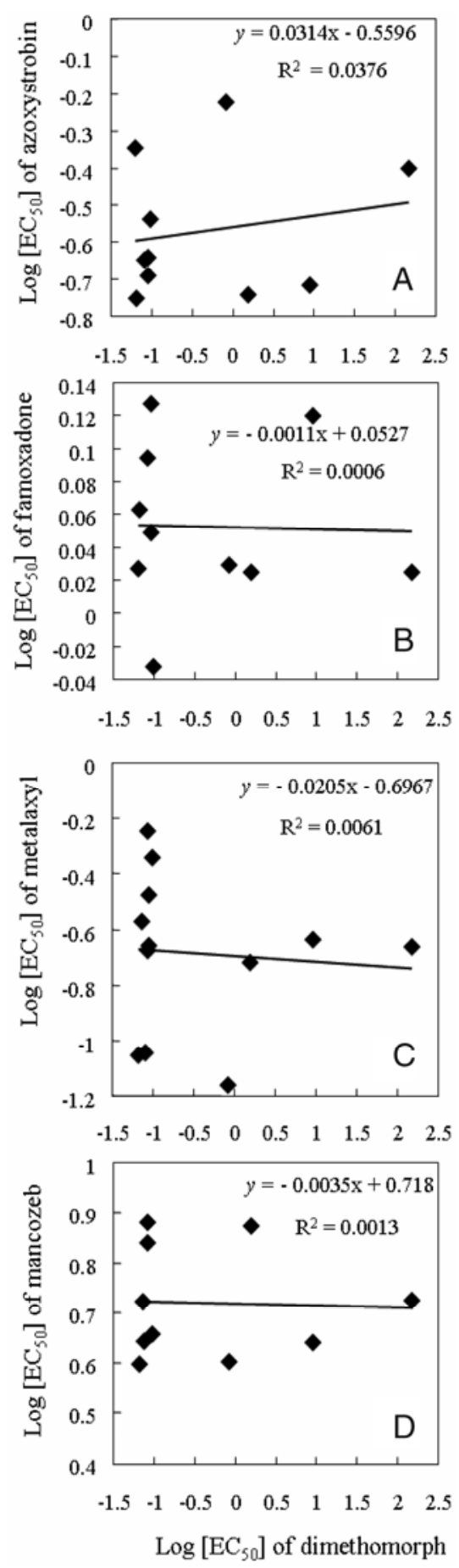

Fig. 2. Correlation between dimethomorph resistance and resistance to the fungicides $\mathbf{A}$, azoxystrobin, $\mathbf{B}$, famoxadone, $\mathbf{C}$, metalaxyl, and $\mathbf{D}$, mancozeb in carboxylic acid amide-reistant and wild-type isolates of Peronophythora litchii. 
values of $\approx 4$ to dimethomorph were obtained through two-step mutagenesis with nitrosomethyl urea on cystospores (2). However, no CAA-resistant isolates of Phytophthora infestans were detected in the field in extensive monitoring studies (as reported by the FRAC CAA Working Group and according to unpublished BASF monitoring studies) and this should be considered in the interpretation of the data.
As noted, CAA resistance in Peronophythora litchii has not been detected in the field. Nonetheless, for a sustainable use of CAA fungicides, the recommendations of the FRAC CAA Working Group should be followed, which limit the number of applications and recommend alternation and combinations with fungicides that have other modes of action to reduce selection pressure and minimize the risk of the occurrence

TABLE 2. Mycelial growth, sporulation, germination, zoospore formation, and pathogenicity on lychee fruits of fungicide-resistant isolates and the corresponding parent isolate of Peronophythora litchii

\begin{tabular}{|c|c|c|c|c|c|c|c|}
\hline \multirow[b]{2}{*}{ Isolate } & \multicolumn{7}{|c|}{ Fitness components ${ }^{\mathrm{s}}$} \\
\hline & $\begin{array}{c}\text { Mycelial } \\
\text { growth }^{\mathrm{t}}(\mathrm{mm})\end{array}$ & $\begin{array}{l}\text { Sporulation in } \\
\text { vitro }^{\mathrm{u}}\left(\text { per } \mathrm{mm}^{2}\right)\end{array}$ & $\begin{array}{c}\text { Sporulation in } \\
\operatorname{vivo}^{v}\left(\text { per } \mathrm{mm}^{2}\right)\end{array}$ & $\begin{array}{c}\text { Sporangia } \\
\text { germination }^{\mathrm{w}}(\%)\end{array}$ & $\begin{array}{c}\text { Cystospore } \\
\text { germination }{ }^{\mathrm{x}}(\%)\end{array}$ & $\begin{array}{c}\text { Zoospore } \\
\text { formation }^{\mathrm{y}}(\%)\end{array}$ & $\begin{array}{c}\text { Diseased } \\
\operatorname{area}^{\mathrm{z}}\left(\mathrm{mm}^{2}\right)\end{array}$ \\
\hline Parent & $66.3 \pm 0.9 b$ & $3,227 \mathrm{a}$ & $1,537 \mathrm{a}$ & $73.3 \mathrm{a}$ & $99.0 \mathrm{a}$ & $93.0 \mathrm{a}$ & $980.1 \mathrm{a}$ \\
\hline Yg6 & $65.7 \pm 1.2 \mathrm{c}$ & $849 \mathrm{~b}$ & $353 \mathrm{c}$ & $53.7 \mathrm{c}$ & $83.0 \mathrm{~b}$ & $69.5 \mathrm{c}$ & $325.8 \mathrm{~b}$ \\
\hline Yg9 & $46.0 \pm 1.4 \mathrm{~d}$ & $3,057 \mathrm{a}$ & $1,283 \mathrm{a}$ & $60.3 \mathrm{~b}$ & $95.8 \mathrm{a}$ & $86.8 \mathrm{~b}$ & $837.7 \mathrm{a}$ \\
\hline Yg6-1 & $80.0 \pm 0 \mathrm{a}$ & $340 \mathrm{~b}$ & $614 b$ & $66.7 \mathrm{~b}$ & $81.0 \mathrm{~b}$ & $63.0 \mathrm{c}$ & $327.4 \mathrm{~b}$ \\
\hline Yg6-3 & $81.3 \pm 0.5 \mathrm{a}$ & $340 \mathrm{~b}$ & $622 \mathrm{~b}$ & $70.33 \mathrm{a}$ & $86.3 \mathrm{~b}$ & $63.0 \mathrm{c}$ & $378.5 \mathrm{~b}$ \\
\hline
\end{tabular}

${ }^{\mathrm{s}}$ Statistical analysis within isolates; means of individual isolates followed by the same letter are not significantly different according to the least significant difference multiple range test $(P=0.05)$.

${ }^{\mathrm{t}}$ Mean colony diameter $(\mathrm{mm})$ measurements after 6 days of incubation $(n=3)$.

${ }^{\mathrm{u}}$ Mean number of sporangia per $\mathrm{mm}^{2}$ of colony after 6 days of incubation $(n=3)$

${ }^{v}$ Mean number of sporangia per $\mathrm{mm}^{2}$ of lesions on litchi fruit $(n=12)$.

${ }^{w}$ Percentage of germinated sporangia after $8 \mathrm{~h}$ incubation $(n=300)$.

x Percentage of germinated cystospores after $4 \mathrm{~h}$ incubation $(n=300)$.

y Percentage of sporangia that released zoospores after $2 \mathrm{~h}$ of incubation $(n=300)$. A sporangium was scored as released if it was empty at evaluation

${ }^{\mathrm{z}}$ Measurements based on the areas of diseased lesions of litchi fruit.

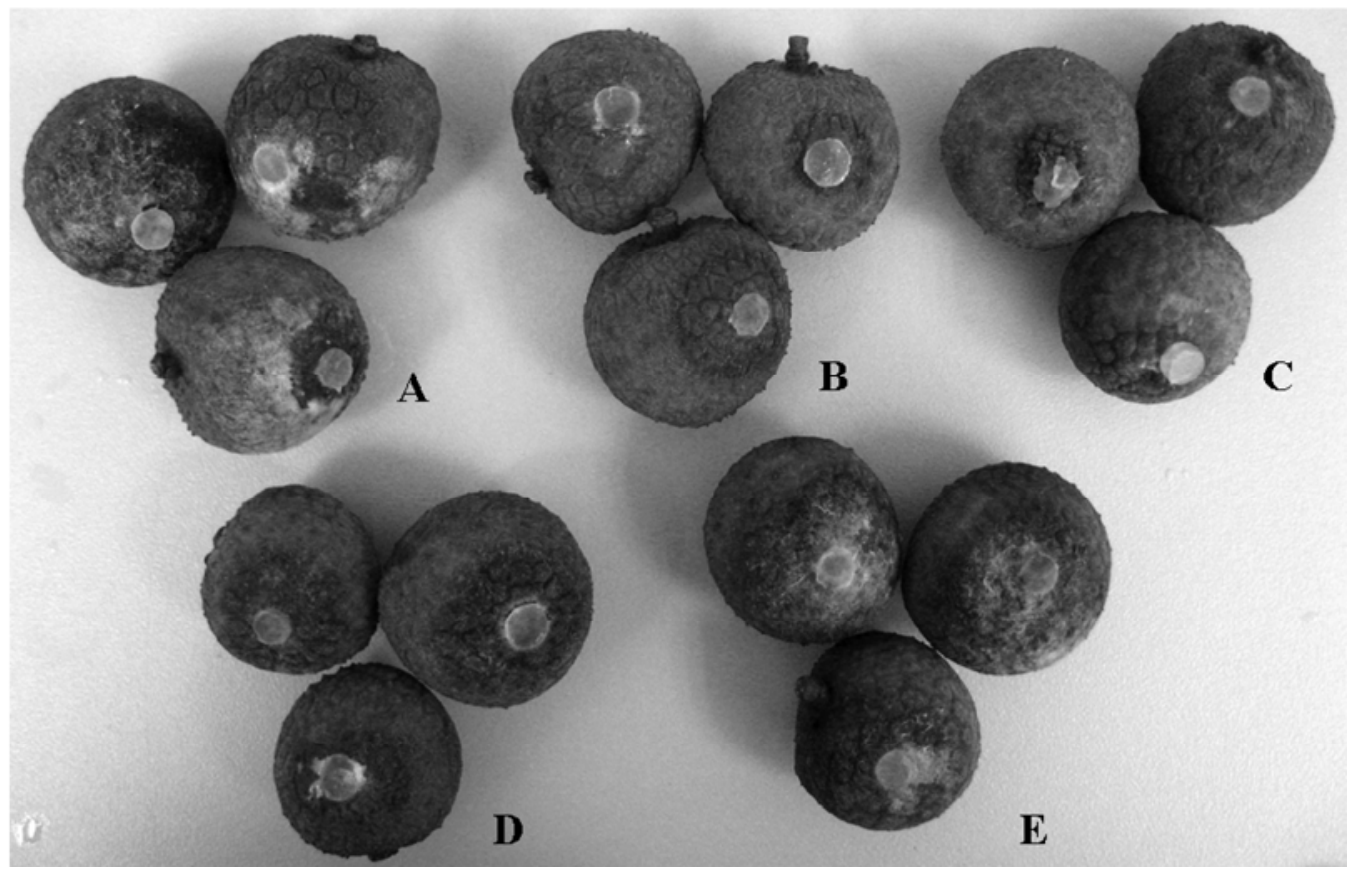

Fig. 3. Comparison of the aggressiveness of carboxylic acid amide-resistant isolates (B, Yg6; C, Yg6-1; D, Yg6-3; and E, Yg9) and A, original wild-type strain of Peronophythora litchii on litchi fruit.

TABLE 3. Colony diameters of resistant and sensitive isolates of Peronophythora litchii at various temperatures on lima bean agar medium

\begin{tabular}{|c|c|c|c|c|c|c|}
\hline \multirow[b]{2}{*}{ Isolate } & \multicolumn{6}{|c|}{ Colony diameter $(\mathrm{mm})^{\mathrm{z}}$} \\
\hline & $10^{\circ} \mathrm{C}$ & $18^{\circ} \mathrm{C}$ & $20^{\circ} \mathrm{C}$ & $25^{\circ} \mathrm{C}$ & $30^{\circ} \mathrm{C}$ & $37^{\circ} \mathrm{C}$ \\
\hline Parent & $50 \mathrm{~b}$ & $53 \mathrm{c}$ & $55 \mathrm{~b}$ & $66 \mathrm{~b}$ & $63 \mathrm{~b}$ & $5 \mathrm{a}$ \\
\hline Yg6 & $45 \mathrm{c}$ & $52 \mathrm{c}$ & $53 \mathrm{~b}$ & $67 \mathrm{~b}$ & $62 \mathrm{~b}$ & $5 \mathrm{a}$ \\
\hline Yg6-3 & $60 \mathrm{a}$ & $62 \mathrm{a}$ & $67 \mathrm{a}$ & $81 \mathrm{a}$ & $70 \mathrm{a}$ & $5 \mathrm{a}$ \\
\hline Yg9 & $27 \mathrm{~d}$ & $43 \mathrm{~d}$ & $45 \mathrm{c}$ & $56 \mathrm{c}$ & $55 \mathrm{c}$ & $5 \mathrm{a}$ \\
\hline
\end{tabular}

${ }^{\mathrm{z}}$ Colony diameters were checked after 37 days of incubation in the dark for the $10^{\circ} \mathrm{C}$ treatment and after 7 days of incubation in the dark for all other temperature treatments. Mean values within a column followed by the same letter are not significantly different according to the least significant difference multiple range test $(P=0.05)$. 
or establishment of resistant individuals. The use recommendations are updated annually and are available on the FRAC homepage.

\section{ACKNOWLEDGMENTS}

This research project was funded by BASF SE. We thank the manufacturers for providing technical-grade fungicides.

\section{LITERATURE CITED}

1. Ann, P. J., and Ko, W. H. 1980. Oospore germination of Peronophythora litchii. Mycologia 72:611-614.

2. Bagirova, S. F., Li, A. Z., Dolgova, A. V., Elansky, S. N., Shaw, D. S., and Dyakov, Y. T. 2001. Mutants of Phytophthora infestans resistant to dimethomorph fungicide. J. Rus. Phytopathol. 2:19-24.

3. Brandt, U., Schagger, H., and Jagow, V. G. 1988. Characterization of binding of the methoxyacrylate inhibitors to mitochondrial cytochrome $\mathrm{c}$ reductase. Eur. J. Biochem. 173:499-506.

4. Caten, C. E., and Jinks, J. L. 1968. Spontaneous variability of single isolates of Phytophthora infestans. I. Cultural variations. Can. J. Bot. 46:329-348.

5. Chabane, K., Lerous, P., and Bompeix, G. 1993. Selection and characterization of Phytophthora parasitica mutants with ultraviolet-induced resistance to dimethomorph or metalaxyl. Pestic. Sci. 39:325-329.

6. Chi, P. K., Pang, X. P., and Liu, R. 1984. On downy blight of litchi chinensis sonn. the pathogen and its infection process. Acta Phytopathol. Sinica. 2:113-119.

7. Cohen, Y., and Baider, A. 1995. Dimethomorph activity against oomycete fungal plant pathogens. Phytopathology 85:1500-1506.

8. Cohen, Y., Rubin, E., Hadad, T., Gotlleb, D., Sierotzki, H., and Gisi, U. 2007. Sensitivity of Phytophthora infestans to mandipropamid and the effect of enforced selection pressure in the field. Plant Pathol. 56:836-842.

9. Dereviagina, M. K., Elanskij, S. N., and Diakov, Y. T. 1999. Resistance of Phytophthora infestans to the dimethomorph fungicide. Mikol. Fitopatol. 33:208-213.

10. Fernandez-Ortuno, D., Tores, J. A., deVincente, A., and Perez-Garcia, A. 2008. Mechanisms of resistance to QoI fungicides in phytopathogenic fungi. Int. Microbiol. 11:1-9.

11. Gisi, U., Waldner, M., Kraus, N., Dubuis, P. H., and Sierotzki, H. 2007. Inheritance of resistance to carboxylic acid amide (CAA) fungicides in Plasmopara viticola. Plant Pathol. 56:199-208.

12. Griffiths, R. G., Dancer, J., O'Neill, E., and Harwood, J. L. 2003. A mandelamide pesticide alters lipid metabolism in Phytophthora infestans. New Phytol. 158:345-353.

13. Hang, X. M. 2001. Lychee production in China. Pages 41-45 in: Lychee Production in the Asia-Pacific Region. M. K. Papademetriou and F. J. Dent, eds. FAO/RAP Publication, Bangkok, Thailand.

14. Koenraadt, H., and Jones, A. L. 1993. Resistance o benomyl conferred by mutations in codon 198 or 200 of the beta-tubulin gene of Neurospora crassa and sensitivity to diethofencarb conferred by codon 198. Phyto- pathology 83:850-854.

15. Kuhn, P. J., Pitt, D., Lee, S. A., Wakley, G., and Sheppard, A. N. 1991. Effects of dimethomorph on the morphology and ultrastructure of Phytophthora. Mycol. Res. 95:333-340.

16. Matheron, M. E., and Porchas, M. 2000. Impact of azoxystrobin, dimethomorph, fluazinam, fosetyl-Al, and metalaxyl on growth, sporulation, and zoospore cyst germination of three Phytophthora spp. Plant Dis. $84: 454-458$

17. Reuveni, M. 2003. Activity of the new fungicide benthiavalicarb against Plasmopara viticola and its efficacy in controlling downy mildew in grapevines. Eur. J. Plant Pathol. 109:243-251.

18. Rubin, E., and Cohen, Y. 2006. An improved method for infecting tomato leaves or seedlings with oospores of Phytophthora infestans used to investigate F1 progeny. Plant Dis. 90:741-749.

19. Rubin, E., Hadad, T., Gisi, U., and Cohen, Y. 2006. Mutagenesis of Phytophthora infestans for resistance against dimethomorph and mefenoxam. (Abstr.) Phytoparasitic 34:305-306.

20. Stammler, G., Carstensen, M., Koch, A., Semar, M., Strobel, S., and Schlehuber, S. 2008. Frequency of different CYP51-haplotypes of Mycosphaerella graminicola and their impact on epoxiconazole-sensitivity and -field efficacy. Crop Prot. 27:1448-1456.

21. Stein, J. M., and Kirk, W. W. 2004. The generation and quantification of resistance to dimethomorph in Phytophthora infestans. Plant Dis. 88:930934.

22. Suty, A., and Stenzel, K. 1999. Iprovalicarb - sensitivity of Phytophthora infestans and Plasmopara viticola: Determination of baseline sensitivity and assessment of the risk of resistance. Pflanzenschutz-Nachrichten Bayer 52:171-182.

23. Voglmayr, H. 2003. Phylogenetic relationships of Peronospora and related genera based on nuclear ribosomal ITS sequences. Mycol. Res. 107:1132-1142.

24. Wang, H. C., Sun, H. Y., Ma, J. X., Stammler, G., and Zhou, M. G. 2009. Fungicide effectiveness during the various developmental stages of Peronophythora litchii in vitro. J. Phytopathol. 157:407-412.

25. Wang, H. C., Sun, H. Y., Stammler, G., Ma, J. X., and Zhou, M. G. 2009. Baseline and differential sensitivity of Peronophythora litchii (lychee downy blight) to three carboxylic acid amide fungicides. Plant Pathol. 58:571-576.

26. Yuan, S. K., Liu, X. L., Si, N. G., Dong, J., Gu, B. G., and Jiang, H. 2006. Sensitivity of Phytophthora infestans to flumorph: In vitro determination of baseline sensitivity and the risk of resistance. Plant Pathol. 55:258-263.

27. Zhang, Z. G., Zheng, X. B., Wang, Y. C., and Ko, W. H. 2007. Evaluation of the rearrangement of taxonomic position of Peronophythora litchii based on partial DNA sequences. Bot. Studies 48:79-89.

28. Zhu, S. S., Liu, X. L., Wang, Y., Wu, X. H., Liu, P. F., Li, J. Q., Yuan, S. K., and Si, N. G. 2007. Resistance of Pseudoperonospora cubensis to flumorph on cucumber in plastic houses. Plant Pathol. 56:967-975.

29. Ziogas, B. N., Markoglou, A. N., Theodosiou, D. I., Anagnostou, A., and Boutopoulou, S. 2006. A high multi-drug resistance to chemically unrelated oomycete fungicides in Phytophthora infestans. Eur. J. Plant Pathol. 115:283-292. 